\title{
Evidence of angular momentum transport in main-sequence solar-like stars
}

\author{
Othman Benomar $^{1} \dagger$, Masao Takata ${ }^{1}$, Hiromoto Shibahashi ${ }^{1}$, \\ Tugdual Ceillier ${ }^{2}$ and Rafael A. García ${ }^{2}$ \\ ${ }^{1}$ Department of Astronomy, School of Science, The University of Tokyo, \\ Bunkyo-ku, Tokyo 113-0033, Japan \\ ${ }^{2}$ Laboratoire AIM, CEA/DSM - CNRS - Univ. Paris Diderot - IRFU/SAp, \\ Centre de Saclay, 91191, Gif-sur-Yvette Cedex, France
}

\begin{abstract}
The rotation rates in the interior and at the surface is determined for the 22 mainsequence stars with masses between 1.0 and $1.6 \mathrm{M}_{\odot}$. The average interior rotation is measured using asteroseismology, while the surface rotation is measured by the spectroscopic $v \sin i$ or the periodic light variation due to surface structures, such as spots. It is found that the difference between the surface rotation rate determined by spectroscopy and the average rotation rate for most of stars is small enough to suggest that an efficient process of angular momentum transport operates during and/or before the main-sequence stage of stars. By comparing the surface rotation rate measured from the light variation with those measured by spectroscopy, we found hints of latitudinal differential rotation. However, this must be confirmed by a further study because our result is sensitive to a few data points.
\end{abstract}

Keywords. methods: data analysis, stars: interiors, stars: oscillations, stars: rotation, stars: low-mass

\section{Introduction}

An important process to understand the stellar rotation is the angular momentum transport. Starting from the pre-main sequence stage, in which uniform rotation is supposed to be realised due to the fully convective structure, the stellar core contracts while the envelope expands in almost all phases of evolution. Therefore, the rotation rate of the core should be larger than that of the envelope, as long as the angular momentum is locally conserved. This could be compensated by the transport of the angular momentum from the core to the envelope. Several observations support the presence of efficient angular momentum transport. For example, helioseismology has shown evidence of nearly-uniform rotation for the Sun in the radial direction, from 30 per cent of the total radius to the surface (e.g. Schou et al. 1998; Thompson et al. 2003; Eff-Darwich \& Korzennik 2013). Almost uniform rotation is also demonstrated in a main-sequence A star (Kurtz et al. 2014) and in a main-sequence F star (Saio et al. 2015). Furthermore, the measured core-to-envelope ratio of the rotation rate of several subgiants and young red giants does not exceed 60 (e.g. Beck et al. 2012; Deheuvels et al. 2012). These studies indicate that a mechanism that transports efficiently the angular momentum from the deep interior to the outer layers could be common in low-mass stars. However, the number of main-sequence stars with a known internal rotation remains limited.

The present work aims to provide a statistical overview on the rotation structure for main-sequence solar-like stars by comparing the average internal rotation with the surface rotation. The average internal rotation rate $f_{\text {seis }}$ is measured by the means of 
asteroseismology, while the surface rotation is determined either by (1) spectroscopy $\left(f_{\text {surf }}^{(1)}\right)$ or by $(2)$ measuring the luminosity modulation due to stellar structure, such as spots at the surface of the $\operatorname{star}\left(f_{\text {surf }}^{(2)}\right)$. In case of differential rotation, $f_{\text {seis }}$ must differ from $f_{\text {surf }}^{(1)}$ and/or $f_{\text {surf }}^{(2)}$.

The comparison was done over 22 main-sequence solar-like stars observed by the CoRoT (Baglin et al. 2006a,b) and Kepler (Borucki et al. 2010) spacecrafts. These were chosen to be rotating approximately 5-10 times faster than the Sun, easing the measure of the rotation. The stars are also of mass between $1.0-1.6 M_{\odot}$ and their evolutionary stages cover the whole main-sequence.

In Section 2 we briefly describe the used methods to determine the rotation. In Section 3 we summarise our results regarding the radial differential rotation, which were already published in Benomar et al. (2015) and also discuss the potential detection of the latitudinal differential rotation.

\section{Principles and method}

This section describes the methods used to derive the interior rotation and the surface rotation of the sampled stars.

\subsection{Internal rotation and stellar inclination}

Asteroseismology has the potential to probe physical process of stellar interior by the means of the global star pulsations detected at the surface. Recent exquisite asteroseismic data gathered by the CoRoT and Kepler spacecrafts revealed oscillations for a few hundreds of main sequence solar-like stars (e.g Appourchaux et al. 2012; Mathur et al. 2012), enabling to measure their internal rotation rates to an unprecedented level of precision. The internal rotation is measured using the so called rotational splitting. Without rotation, pulsation eigenfrequencies are specified by two integers: the radial order $n$ and the degree $l$ of the modes. However, similarly to the Zeeman effect observed in atomic spectral lines in presence of a magnetic field, the rotation lifts the degeneracy among non-radial pulsation modes with a given $n$ and $l(l>0)$ so that a discrete structure of modes appears, whose componnent is identified by the azimuthal order $m$. In a slow rotator, the effect of rotation can be analysed in the framework of linear perturbation theory, so that each mode frequency $\nu_{n, l, m}$ is given by,

$$
\nu_{n, l, m}=\nu_{n, l}+m \delta \nu_{n, l} .
$$

Here, $\nu_{n, l}$ is the frequency for a spherically symmetric star, while $\delta \nu_{n, l}$ is the rotational splitting. For the observed modes of the stars in our sample, $\delta \nu_{n, l}$ is approximately $30-60 \%$ sensitive to the rotation within the deep radiative zone, with this strongly depending on the stellar mass. Because the massive stars have a thinner convective zones than less massive stars, the sensitivity to the rotation of the radiative zone is greater in the more massive stars. This is well illustrated by figure 1 in Benomar et al. (2015). Furthermore, the observed low degree $(l<4)$ and high order $(n \gtrsim 10)$ acoustic modes propagate in roughly in the same region, so that they are almost sensitive to the same layers. As a consequence, $\delta \nu_{n, l}$ can be assumed to be equal to an average rotation rate in the interior, which is represented by $f_{\text {seis }}$.

Due to the turbulent convection, which is the source of excitation of the acoustic modes, each solar-like mode has a Lorentzian profile in the power spectrum (Harvey 1985). Thus the power spectrum of the stellar oscillations can be expressed as a sum of Lorentzians over $n, l$ and $m$ and characterised by a height $H_{n, l, m}$ and a width at half maximum 
$\Gamma_{n, l, m}$

$$
P(\nu)=\sum_{n, l} \sum_{m=-l}^{l} \frac{H_{n, l, m}}{1+4\left(\nu-\nu_{n, l, m}\right)^{2} / \Gamma_{n, l, m}^{2}} .
$$

In disk integrated photometry, the height of the split modes depend on the stellar inclination angle $i$ as $H_{n, l, m}=\mathcal{E}_{l, m}(i) H_{n, l}$ (Ballot, García \& Lambert 2006; Gizon \& Solanki 2003). Here $H_{n, l}$ is the intrinsic height for the mode, while $\mathcal{E}_{l, m}(i)$ is the visibility of the $m$-components in the power spectrum,

$$
\mathcal{E}_{l, m}(i)=\frac{(l-|m|) !}{(l+|m|) !}\left[P_{l}^{|m|}(\cos i)\right]^{2},
$$

with $P_{l}^{|m|}$ is the associated Legendre function. As it is evident here, $\mathcal{E}_{l, m}(i)$ can be used to measure the stellar inclination, which is important to derive the surface rotation rate from the spectroscopic $v \sin i$.

The probability density function for the rotational splitting and the stellar inclination, as well as their correlation is determined by fitting equation (2.2) to the power spectrum and based on a Markov Chain Monte Carlo algorithm, presented in Benomar, Appourchaux \& Baudin (2009).

\subsection{Surface rotation}

Two methods are used to determine the surface rotation. The first method uses the rotational broadening of spectroscopic absorption lines, which gives $v \sin i=2 \pi R f_{\operatorname{surf}}^{(1)} \sin i$. Here, $R$ corresponds to the stellar radius. The measurement of $v \sin i$ for the stars in our sample was done by several publications (Marcy et al. 2014; Bruntt 2009; Bruntt et al. 2012; Pál et al. 2008). More details can be found in Benomar et al. (2015). The second method measures the periodicity $P_{\text {rot }}=1 / f_{\text {surf }}^{(2)}$ of the luminosity modulation due to stellar structures at the surface of the star attributed to surface spots. These were measured by Benomar, Appourchaux \& Baudin (2009) and Barban et al. (2009) for the CoRoT stars, and by García et al. (2014) for the Kepler stars.

Note that $f_{\text {surf }}^{(1)}$ and $f_{\text {surf }}^{(2)}$ may not be sensitive to the surface rotation of the same regions. The rotational broadening is determined by contributions from a wide range of the area on the visible hemisphere, so that an average of the rotation over the visible stellar disk is measured. On the other hand, the brightness modulation is sensitive to the rotation period at the latitude where transient spots evolve. In the case of the Sun, spots migrate from $\approx 30^{\circ}$ (Maunder 1904) toward the equator during their 11-year cycle. Thus, assuming that the Sun is a representative solar-like star, the observed rotation frequency $f_{\text {surf }}^{(1)}$ and $f_{\text {surf }}^{(2)}$ should differ in case of significant latitudinal differential rotation.

\subsection{Theoretical rotationnal splitting}

In order to evaluate the significance of our result, we determined the theoretical average rotational splitting in absence of angular momentum transport between the interior and surface of stars. For achieving this, the evolution of the rotation profiles of 1.2 and $1.5 \mathrm{M}_{\odot}$ stellar models with the solar composition has been calculated (H. Saio, private communication) from the (pre-main-sequence) Hayashi phase to the Zero-Age Main-Sequence (ZAMS) stage, with the following assumptions: (1) rotation rates are slow enough to have negligible effects on the structure, which keeps spherical symmetry, (2) total angular momentum is conserved (no addition to and no removal from the surface layers are considered), (3) the rotation profiles are functions of only radius, (4) angular momentum is locally conserved in the radiative zone and (5) it is redistributed instantaneously in 

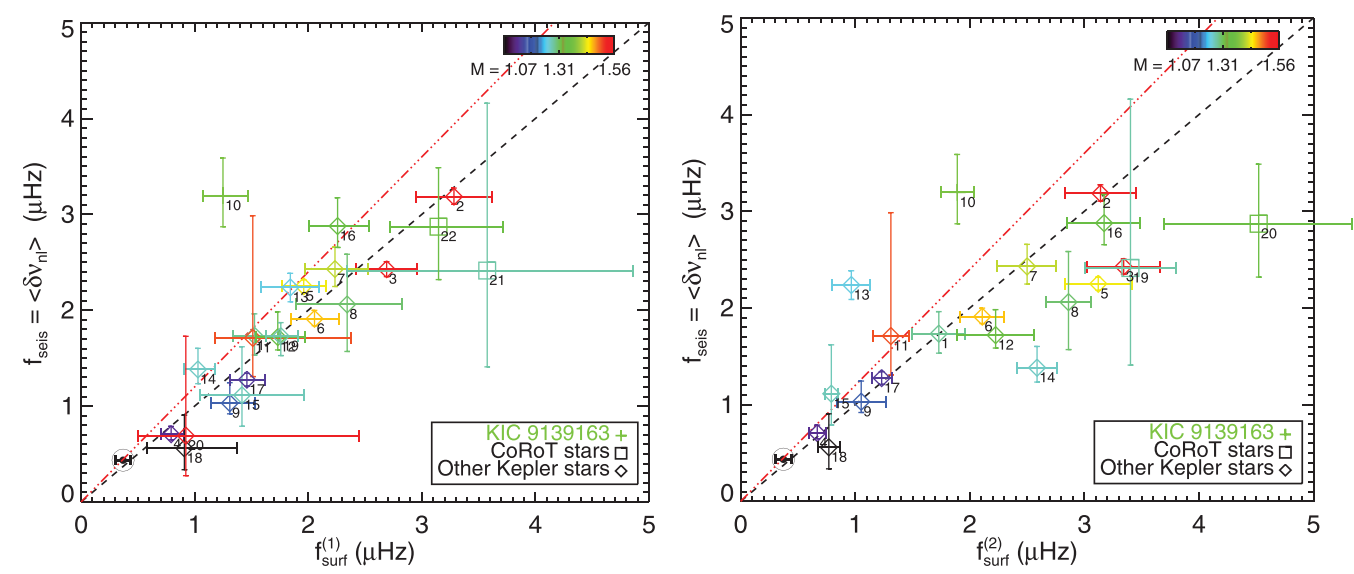

Figure 1. The rotation from seismology compared with the surface rotation from spectroscopic $v \sin i$ - left, reproduced from Benomar et al. (2015) — or from the lightcurve modulation due to spot - right -

the convection zone to establish a constant rotation rate (a limiting case of the efficient transport) with the total angular momentum of the zone fixed.

During the evolution after the ZAMS, the central mass concentration increases so that spinning-up of deep layers is expected in absence of angular momentum transport. Therefore, the evaluated ratio between the internal and surface rotation rates corresponds to a lower limit of the expected rotation rate within the main-sequence. This is explained in further details in Benomar et al. (2015).

\section{Results}

Fig. 1 compares the rotation rates from seismology $f_{\text {seis }}$ with the surface rotation $f_{\text {surf }}^{(1)}$ (left) or $f_{\text {surf }}^{(2)}$ (right). The Sun-as-a-star is also shown as a reference and the stars have the same indices as in Benomar et al. (2015). The red dashed triple-dotted line shows the theoretical rotational splitting as a function of the surface rotation rate. We note that most of the observations lie below the red line, using either $f_{\text {surf }}^{(1)}$ or $f_{\text {surf }}^{(2)}$. This indicates that for the majority of the stars, the rotation in the deep layers is slower than expected by a model that does not include angular momentum transport. We conclude that a mechanism of transport of angular momentum is required in most of the stars.

By comparing the two figures we note discrepancies between $f_{\text {surf }}^{(1)}$ and $f_{\text {surf }}^{(2)}$ of up to a factor two. It is unlikely that a bias arises from the macroscopic/microscopic turbulence because it is expected to affect by only a few percent the measure of $v \sin i$.

However, the discrepancy could arise from the fact in some stars, multiple spots appear at the same time at the surface of the stars whereas the spot method is accurate only if one main structure exists.

The last hypothesis is that the intrinsic difference between the two estimates of the surface rotation originates from a latitudinal differential rotation. We should note however, that this could not explain the case of star 10 , for which $f_{\text {surf }}^{(1)}$ and $f_{\text {surf }}^{(2)}$ are consistent within 2 sigma to each other, but inconsistent with $f_{\text {seis }}$. From our observation, this is actually the only unambiguous candidate for a star with an important radial differential rotation.

To identify whether a latitudinal differential rotation could explain the apparent discrepancy between $f_{\text {surf }}^{(1)}$ and $f_{\text {surf }}^{(2)}$ for the other stars, we plot in Fig. 2 the ratio $f_{\text {surf }}^{(1)} / f_{\text {surf }}^{(2)}$ 


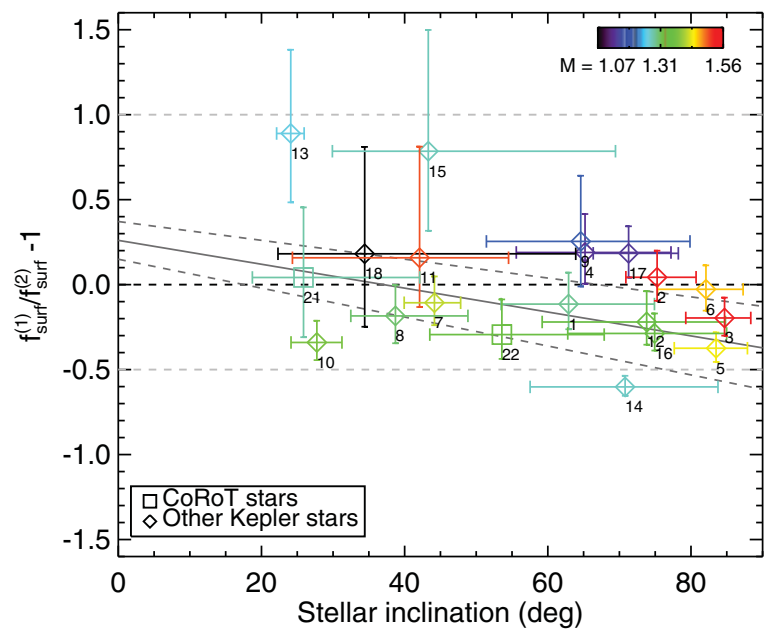

Figure 2. Dependence of the ratio between the two measurements of the surface rotation on the stellar inclination $i$, derived from spectroscopic $v \sin i\left(f_{\text {surf }}^{(1)}\right)$ and the lightcurve modulation due to spot $\left(f_{\text {surf }}^{(2)}\right)$. The solid gray line is the best fit a linear function. The uncertainties at $1 \sigma$ are shown with the oblique dashed lines. The horizontal gray dashed lines indicate the ratios of two and of a half. Reproduced from Benomar et al. (2015).

as a function of the stellar inclination $i$. In the Sun, the surface rotation in the polar region $\left(i \approx 0^{\circ}\right)$ is $25 \%$ slower than the radiative zone (which rotates uniformly). However, it is $7 \%$ faster at the equator $\left(i \approx 90^{\circ}\right)$ than the radiative zone. It is considered that stellar spots appear preferably around the equator, as in the case of the Sun, so that if the Sun is a representative solar-like star, a trend should be visible in Figure 2 . Interestingly this figure indeed exhibits a decreasing trend as a function of the stellar inclination, qualitatively consistent with a slower pole than the equator. To assess the trend significance, we fitted a linear function $f_{\text {surf }}^{(1)} / f_{\text {surf }}^{(2)}=1+a-b i$. We obtain the ordinate at origin $a=0.26 \pm 0.12$ and the slope $b=0.0015 \pm 0.008$. While $b$ is significant (at a $1 \sigma$ level), the result is sensitive to a few extreme observations. For example, by removing the star 14, the slope is not anymore significant. A study on a larger sample might allow to better determine the relevance of our current observation.

\section{Acknowledgement}

We thank NASA and CNES, as well as the Kepler and CoRoT teams for their revolutionary data. O.B. is supported by Japan Society for Promotion of Science (JSPS) Fellowship for Research (No. 25-13316). M.T. thanks the support by JSPS KAKENHI Grant Number 26400219. R.A.G. and T.C. thank the support of the CNES.

\section{References}

Appourchaux, T., et al., 2012, A\&SA, 543, A54

Baglin, A., Auvergne, M., Barge, P., Deleuil, M., Catala, C., Michel, E., \& Weiss, W., COROT Team, 2006a, in ESA Special Publication, Vol. 1306, ESA Special Publication, Fridlund, M., Baglin, A., Lochard, J., Conroy, L., eds., p. 33

Baglin, A., et al., 2006b, in COSPAR Meeting, Vol. 36, 36th COSPAR Scientific Assembly, p. 3749

Ballot, J., García R. A., \& Lambert, P., 2006, MNRAS, 369, 1281

Barban, C., et al., 2009, A\&A, 506, 51 
Beck, P. G., et al., 2012, Nature, 481, 55

Benomar, O., Appourchaux, T., \& Baudin, F., 2009, A\&A, 506, 15

Benomar, O., Takata, M., Shibahashi, H., Ceillier, T., \& García R. A., 2015, MNRAS, 452, 2654

Borucki, W. J., et al., 2010, Science, 327, 977

Bruntt, H., 2009, A\& A, 506, 235

Bruntt, H., et al., 2012, MNRAS, 423, 122

Deheuvels, S., et al., 2012, ApJ, 756, 19

Eff-Darwich A. \& Korzennik, S. G., 2013, Sol. Phys., 287, 43

García R. A., et al., 2014, A\&A A, 572, A34

Gizon, L. \& Solanki, S. K., 2003, ApJ, 589, 1009

Harvey, J., 1985, ESA SP, 235, 199

Kurtz, D. W., Saio, H., Takata, M., Shibahashi, H., Murphy, S. J., \& Sekii, T., 2014, MNRAS, 444,102

Marcy, G. W., et al., 2014, ApJS, 210, 20

Mathur, S., et al., 2012, ApJ, 749, 152

Maunder, E. W., 1904, MNRAS, 64, 747

Pál A., et al., 2008, ApJ, 680, 1450 (P08)

Saio, H., Kurtz, D. W., Takata, M., Shibahashi, H., Murphy, S. J., Sekii, T., \& Bedding, T. R., 2015, MNRAS, 447, 3264

Schou, J., et al., 1998, ApJ, 505, 390

Thompson, M. J., Christensen-Dalsgaard J., Miesch, M. S., \& Toomre, J., 2003, ARAA, 41, 599 